\title{
An upper bound solution for closed die sinter forging of hexagonal shapes
}

\author{
R K RANJAN $^{1}$ and S KUMAR 2 \\ ${ }^{1}$ Department of Applied Mechanics and \\ ${ }^{2}$ Department of Production Engineering, Birla Institute of Technology, Mesra, \\ Ranchi 835 215, India \\ e-mail: rkranjanbit@yahoo.com
}

MS received 9 May 2003; revised 5 August 2003

\begin{abstract}
The paper reports on an investigation into the various aspects of closed die cold forging of hexagonal powder preforms, which have been compacted and sintered from atomized powder. It is found that for certain dimensional ratios of the preform, the die pressure is minimum. An attempt has been made determine the die pressures developed during the closed die forging of the hexagonal powder preform by using an upper bound approach. The results so obtained are discussed critically to illustrate the interaction of various process parameters involved and are presented graphically.
\end{abstract}

Keywords. Hexagonal powder preform, closed die; sintering.

\section{Introduction}

During the last few years metal powder components have assumed an important position in industry, as they are used successfully in a wide range of applications. Both the mechanical and the metallurgical properties of the metal powder components compare favorably with those of wrought materials (Ramakrishnan 1980). Bulk processing of metal powder preforms is a convenient method of reducing or eliminating the porosity from conventional powder metallurgy products. The process is attractive because it avoids a large number of operations, and the high scrap losses and high-energy consumption associated with conventional manufacturing processes such as casting, machining etc. In this new technology, sintered porous powder preforms are used as starting materials in metal-forming processes. Metal powder products manufactured by this new technology are comparable and in some cases even superior to those of cast and wrought products.

Although a considerable amount of work has been reported recently as the various technological aspects of the industrial processing of metal-powder preforms (Jha \& Kumar 1983, 1984, 1985), no systematic attempt has been made so far to study the processing load and deformation characteristics during forging of hexagonal disc in a closed die.

A list of symbols is given at the end of the paper 
It is expected that the present work will be of great importance for the assessment of die load during the forging of metal powder preforms.

\section{Interfacial friction law}

In investigation of the plastic deformation of metal-powder preforms, it is evident that with the application of compressive hydrostatic stress the pores close and the relative density increases, whereas with the application of tensile hydrostatic stress the pores grow and the relative density decreases. Also, the density distribution does not seem to be uniform throughout, being high in the central region and low at the edges. The density distribution is more uniform for smaller coefficients of friction, $\mu$ and for greater initial density (Tabata et al 1980).

Friction conditions between deforming tool and work piece in metal-forming are of the greatest importance with respect to a number of factors such as force and mode of deformation, properties of the finished specimen and resulting surface roughness. During the sinter forging process, it is very important to pay special consideration to interfacial friction, as this determines the success or failure of the operation. The relative velocity between the work piece material and the die surface, together with high interfacial pressure and/or deformation modes, creates a condition of composite friction, which is due to adhesion and sliding (Deryagin 1952). The shear equation becomes:

$$
\tau=\mu\left[p+\rho_{0} \phi_{0}\right],
$$

the first term $\mu p$ is due to sliding and the second term $\mu \rho_{0} \phi_{0}$ is due to adhesion, which arises from the change of the relative density of the preform during the process.

\section{Plastic deformation of sintered preform}

In investigation of the plastic deformation of sintered deformation of sintered metal powder preforms, it is clear that change in volume occurs due to porosity. A preform with high relative density yields at a relative by high stress, whereas one with low relative density yields at relatively low stress. Even hydrostatic stress can cause sintered metal powder preforms to yield, as the yield surface is closed on the hydrostatic stress axis. The density distribution also does not seem to be uniform throughout, being high in the central region and low at the edges. Density distribution is more uniform for preforms with smaller coefficients of friction and higher initial densities.

Tabata et al (1977) proposed the following yield criterion for porous metal powder preforms:

$$
\rho^{k}=\sqrt{3 J_{2}^{\prime}} \pm 3 \eta \sigma_{m}
$$

The negative sign is taken for $\sigma_{m} \leq 0$ and the positive sign is taken for $\sigma_{m}<0$.

Values of $\eta$ and $k$ were determined experimentally from simple compression and tension test of sintered copper-powder preforms (Tabata \& Masaki 1977) as

$$
\begin{aligned}
& \eta=0.54(1-\rho)^{1 \cdot 2}, \text { for } \sigma_{m} \leq 0, \\
& \eta=0.55(1-\rho)^{0.83}, \text { for } \sigma_{m}<0 \text { and } k=2 .
\end{aligned}
$$


For the axisymmetric condition the yield criterion reduces to

$$
\sigma_{1}=\frac{\rho^{k} \sigma_{0}}{(1-2 \eta)}+\frac{(1+\eta)}{(1-2 \eta)} \sigma_{2}
$$

whilst for the axisymmetric condition the compactibility equation becomes

$$
\varepsilon_{r}=\frac{(2 \eta-1)}{2(1+\eta)} \ln \frac{t_{2}}{t_{1}}, \text { (appendix A). }
$$

\section{Velocity field and strain rate}

\subsection{Velocity field}

By drawing normals to the sides of the hexagon from the in-centre, the disc is divided into six regions, which are symmetrical as far as flow is concerned. In the region GAHO (figure 1) and so also in the other five regions, we assume that during compression the horizontal component of velocity of all particles is directed towards the corner. Taking the corner as the origin of cylindrical coordinates, the velocity components $U_{\theta}$ and $U_{z}$ are as given below:

$$
\begin{aligned}
U_{\theta} & =0, \\
U_{Z} & =(U / t) z .
\end{aligned}
$$
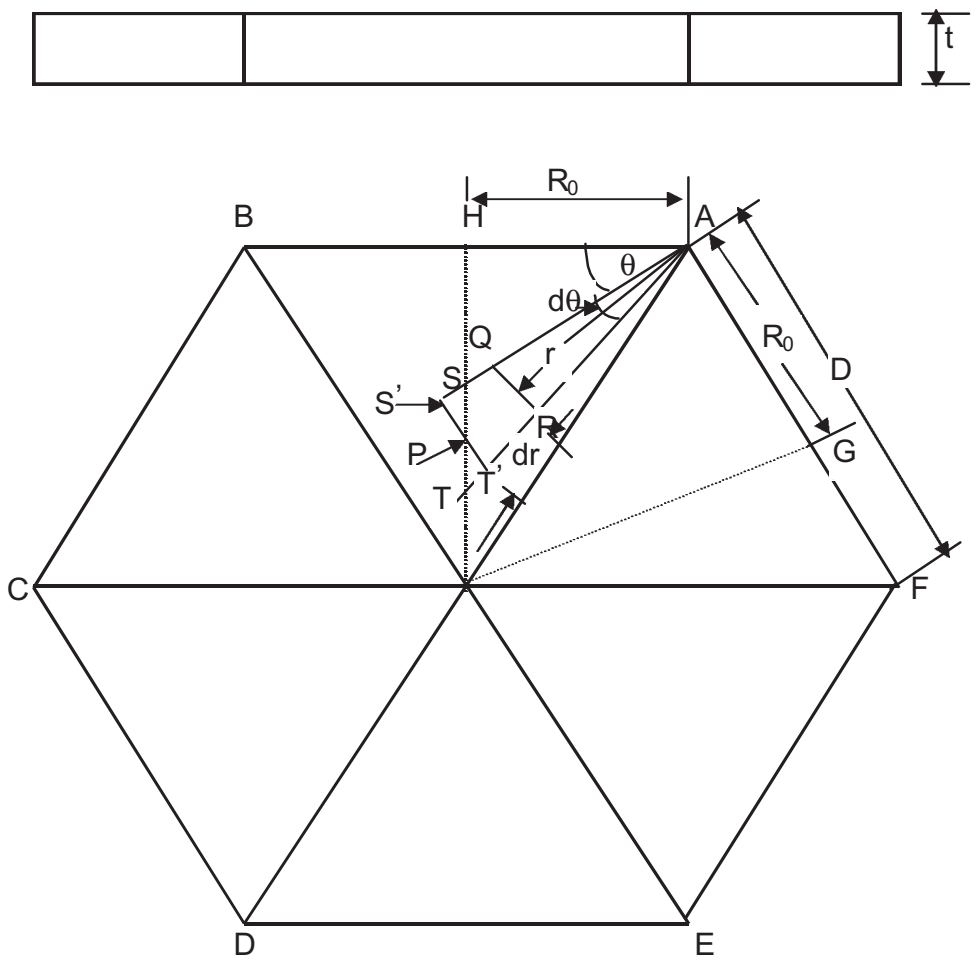

Figure 1. The hexagonal shaped disc with thickness $t$ and side $2 R_{0}$. 
In the deformation of the powder preform, the volume constancy has not been assumed. During the forging of the powder preform, the pores get closer and the volume continuously changes. Hence, in this case, mass constancy of the preform is to be assumed and thus the normal-strain components must satisfy the following compressibility equation for porous materials (Tabata \& Masaki 1975):

$$
\dot{\varepsilon}_{r}+\dot{\varepsilon}_{\theta}+\frac{(1-2 \eta)}{2(1+\eta)} \dot{\varepsilon}_{z}=0 .
$$

From the mass continuity of material flow, it is easily inferred that the rate of flow across section $\mathrm{QR}$ (figure 1) towards the apex $\mathrm{A}$ is equal to the rate at which the material is compressed over the area QRTS. The area T'TP and S'SP being equal, (S'T' is the arc with centre at A) we may take the rate of compression over area QRT'S' instead of area QRTS to simplify the analysis without any error.

Area of the element QRTS is given by

$$
\frac{1}{2}\left(\frac{R_{0}}{\cos \theta} \mathrm{d} \theta+r \mathrm{~d} \theta\right)\left(\frac{R_{0}}{\cos \theta}-r\right)=\frac{1}{2}\left(\frac{R_{0}^{2}}{\cos ^{2} \theta}-r^{2}\right) \mathrm{d} \theta .
$$

Equating the mass flow across the section QR to the rate of pressing over the area QRT'S', (considering (4)) we get:

$$
U_{r}=\frac{(1-2 \eta) U}{2(1+\eta) r t}\left(\frac{R_{0}^{2}}{\cos ^{2} \theta}-r^{2}\right) .
$$

The velocity field given by (3), (4) and (6) must satisfy the boundary conditions viz.

$$
U_{r}=0 \text { at } r=R_{0} / \cos \theta .
$$

\subsection{Strain rate}

At the top surface $U_{Z}=-U=$ velocity of ram. On the side surface, the flow is only along the surface towards the respective corner (apex). The strain rate field is obtained as:

$$
\begin{aligned}
\dot{\varepsilon}_{r} & =\frac{(1-2 \eta) U}{2(1+\eta) t}\left(\frac{R_{0}^{2}}{r^{2} \cos ^{2} \theta}+1\right), \\
\dot{\varepsilon}_{\theta} & =\frac{(1-2 \eta) U}{2(1+\eta) t}\left(\frac{R_{0}^{2}}{r^{2} \cos ^{2} \theta}-1\right), \\
\dot{\varepsilon}_{z} & =-U / t, \\
\dot{\varepsilon}_{r \theta} & =\frac{(1-2 \eta) U}{2(1+\eta) t}\left(\frac{R_{0}^{2}}{r^{2}} \sec ^{2} \theta \cdot \tan \theta\right)
\end{aligned}
$$

The above strain rates satisfy the compressibility equation (5) for powder preforms.

\section{Die load}

For plastic deformation of a metal powder the external power $\stackrel{*}{J}$ supplied by the platens is given as

$$
\stackrel{*}{J}=W_{i}+W_{f}+W_{a}+W_{t} .
$$


The first term on the right hand side denotes the rate of internal energy dissipation $W_{i}$, the second term denotes the frictional shear energy losses $W_{f}$, the third term denotes the energy dissipation due to inertia forces $W_{a}$, and the last term covers power supplied by predetermined body tractions $W_{t}$. In this case forces due to inertia is negligibly small and no external surface traction is stipulated. Therefore, $W_{a}=W_{t}=0$.

Now the external power $\stackrel{*}{J}$ supplied by the press for the entire hexagonal shape through the platen is

$$
\stackrel{*}{J}=\int F_{i} U_{i} \mathrm{~d} s=6 P U=6 p_{a v} U R_{0}^{2} \tan (\pi / 3)=W_{i}+W_{f 1}+W_{f 2} ;
$$

where $W_{f 1}$ is the rate of energy dissipation at the bottom and top of the preform and $W_{f 2}$ is the rate of energy dissipation at the six flat faces of the preform.

\subsection{Rate of dissipation of deformation energy $\left(W_{i}\right)$}

$$
i W_{i}=\frac{2}{\sqrt{3}} \sigma_{i 0}^{*} \int_{v}\left[\frac{1}{2}\left(\dot{\varepsilon}_{r}^{2}+\dot{\varepsilon}_{\theta}^{2}+\dot{\varepsilon}_{z}^{2}+\dot{\varepsilon}_{r \theta}^{2}\right)\right]^{\frac{1}{2}} \mathrm{~d} V .
$$

Simplification of yield criterion for axisymmetric condition gives

$$
\sigma_{0}^{*}=\frac{\rho^{k} \sigma_{0}}{(1-2 \eta)} ; \text { and } \mathrm{d} v=t \cdot r \mathrm{~d} \theta \cdot \mathrm{d} r .
$$

After putting in the values of strain rate from (7)-(10), we get (for all six faces),

$$
W_{i}=\left.6 \cdot \frac{4 \rho^{k} \sigma_{0} U C}{\sqrt{3} \cdot \sqrt{2}(1-2 \eta)} \int_{0}^{\frac{\pi}{3}}\left[\sec ^{3} \theta \cdot \ln r\left(1-\frac{\cos ^{2} \theta}{2}\right)\right]^{\frac{1}{2}}\right|_{0 \cdot 1} ^{R_{0} / \cos \theta} \mathrm{d} \theta .
$$

The lower limit for $r$ is taken as $r_{0}=0 \cdot 1$ to facilitate the calculations. It is verified that there is very little difference in the value of $W_{i}$ for $r_{0}=0 \cdot 1$ to $r_{0}=0.75$.

$$
W_{i}=6 \cdot \sqrt{\frac{2}{3}} \frac{\rho^{k} \sigma_{0} U C R_{0}^{2}}{(1-2 \eta)} A,
$$

where

$$
A=\left[\left(\ln \frac{R_{0}}{\cos \theta}-\ln 0 \cdot 1\right) \sqrt{1-\frac{\cos ^{2} \theta}{2}}\{\tan \theta \cdot \sec \theta+\ln (\sec \theta+\tan \theta)\}\right]_{0}^{\frac{\pi}{3}} .
$$

5.2 Frictional dissipation on the bottom and top surfaces $\left(W_{f 1}\right)$

$$
W_{f 1}=\int_{S} \tau|\Delta v| \mathrm{d} s
$$


where

$$
\begin{aligned}
\tau & =\mu\left[p+\rho_{0} \phi_{0}\right] ;|\Delta v|=\frac{(1-2 \eta) U}{2(1+\eta) r t}\left(\frac{R_{0}^{2}}{\cos ^{2} \theta}-r^{2}\right), \\
W_{f 1} & =12 \cdot \frac{U(1-2 \eta) \mu\left(p+\rho_{0} \phi_{0}\right)}{2(1+\eta)} \iint_{0}^{\frac{\pi R_{0}}{3 \cos \theta}}\left(R_{0}^{2} \sec ^{2} \theta-r^{2}\right) \mathrm{d} \theta \cdot \mathrm{d} r \\
W_{f 1} & =12 \cdot \frac{U(1-2 \eta) \mu\left(p+\rho_{0} \phi_{0}\right) R_{0}^{3}}{2(1+\eta)}\left[\begin{array}{l}
\frac{1}{3}|\tan \theta \cdot \sec \theta+\ln (\sec \theta+\tan \theta)|_{0}^{\frac{\pi}{3}} \\
-\left|\frac{0 \cdot 1 \tan \theta}{R_{0}}\right|_{0}^{\frac{\pi}{3}}+|\theta|_{0}^{\frac{\pi}{3}} \cdot \frac{0 \cdot 001}{3 R_{0}^{3}}
\end{array}\right] .
\end{aligned}
$$

5.3 Frictional dissipation at the six flat surfaces $\left(W_{f 2}\right)$

$$
\begin{aligned}
& W_{f 2}=6 \int_{0}^{t} \tau\left|U_{z}\right| \cdot 2 R_{0} \cdot \mathrm{d} z, \\
& W_{f 2}=\frac{12 U \mu R_{0}}{t}\left(p+\rho_{0} \phi_{0}\right)\left|\frac{z^{2}}{2}\right|_{0}^{t}, \\
& W_{f 2}=6 . U R_{0} \mu t\left(p+\rho_{0} \phi_{0}\right) .
\end{aligned}
$$

Now putting in the values of $W_{i}, W_{f 1}$ and $W_{f 2}$ from (14), (16) \& (17) respectively in (12), we get:

$$
\begin{aligned}
& \frac{p_{a v}}{\sigma_{0}}=\left[\begin{array}{l}
1-\frac{(1-2 \eta) \mu(1+x) R_{0}}{\tan (\pi / 3) \cdot t)} \\
-\frac{\mu t(1+x)}{\tan (\pi / 3) \cdot R_{0}}
\end{array}\left\{\begin{array}{l}
\frac{1}{3}|\tan \theta \cdot \sec \theta+\ln (\sec \theta+\tan \theta)|_{0}^{\frac{\pi}{3}} \\
-\left|\frac{0 \cdot 1 \tan \theta}{R_{0}}\right|_{0}^{\frac{\pi}{3}}+|\theta|_{0}^{\frac{\pi}{3}} \cdot \frac{0 \cdot 001}{3 R_{0}^{3}}
\end{array}\right\}\right]^{-1} \\
& \times \sqrt{\frac{2}{3}} \frac{\rho^{k} \cdot \sigma_{0}}{(1-2 \eta) \cdot \tan (\pi / 3)} .
\end{aligned}
$$

\section{Results and discussion}

For the solution for (18), we have to make two deviations. First is to take the lower limit for $r$ the generic radius as equal to $0 \cdot 1$ instead of zero. This is done to facilitate the calculation of $W_{i}$ and hence enable the feasible solution for $p_{a v} / \sigma_{0}$. If we take $r$ equal to zero then $\log (0)$ becomes minus infinity. This lower limit for $r$ does not impair the solution of the problem, as there is little difference in the value of $W_{i}$ when we take lower limit equal to $0.25,0.5$ and $0 \cdot 75$, particularly for the conditions of lubrication prevailing in actual processing. The second 


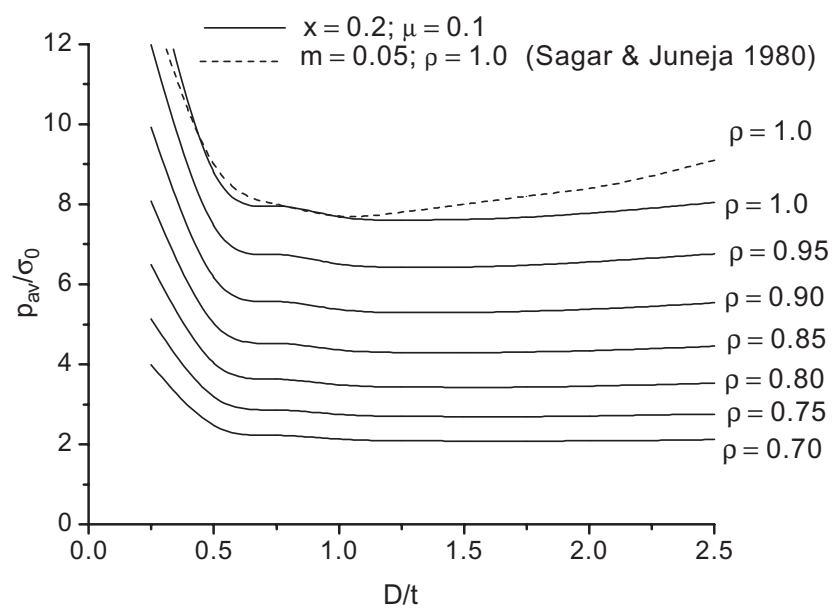

Figure 2. Variation of relative average pressure with $D / t$ for different relative densities of the preform.

deviation is that the curve made by the corner radius is concave if we look from the centre of the hexagon but since we have taken the corner as origin for the study of flow, the curve is convex. Again there is negligibly small difference from the actual case (as the preform does not completely fill up the corners). A certain radius is always there near the corners of the hexagonal disc.

The relative forging pressure for different value of initial relative density shows minima and hence there is an optimum value of $D / t$ (figure 2). The graph for wrought material, proposed by Sagar \& Juneja (1980) is also superimposed on figure 2. A small deviation is seen; it is due to fact that during the present investigation a composite interfacial friction law has been considered. But in the analysis of Sagar \& Juneja (1980), a constant friction factor $(m)$ has been assumed. The optimum value of $D / t$ is of course different at conditions of lubrication (figure 3), i.e. they depend upon the coefficient of factors $\mu$. We see that the minima occur at $D / t$ nearly equal to $0 \cdot 6$. The relative forging pressure increases with increasing value of coefficient of friction. The relative forging pressure increases with percentage reduction in height of preform (figure 4). Again, it increases with increasing value of $D / t$ (figure 5).

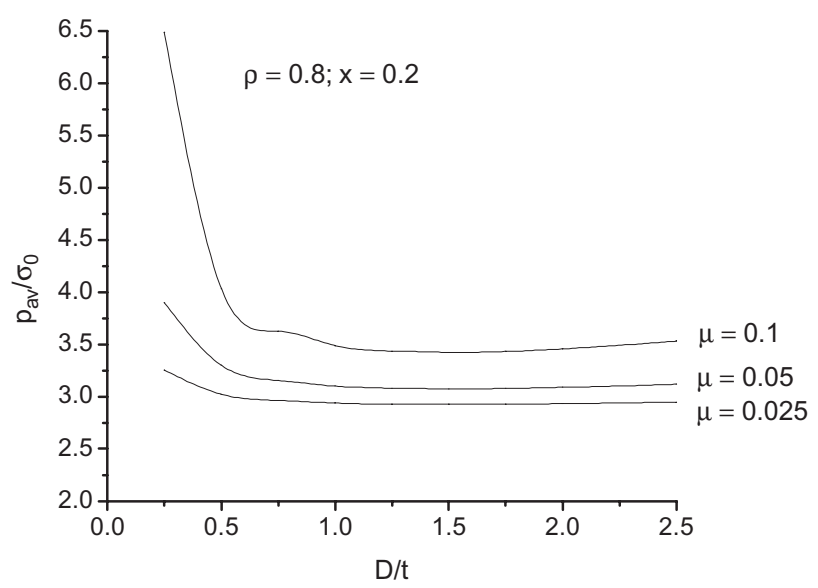

Figure 3. Effect of the condition of lubrication on the relative average pressure for various geometries of the hexagonal disc. 


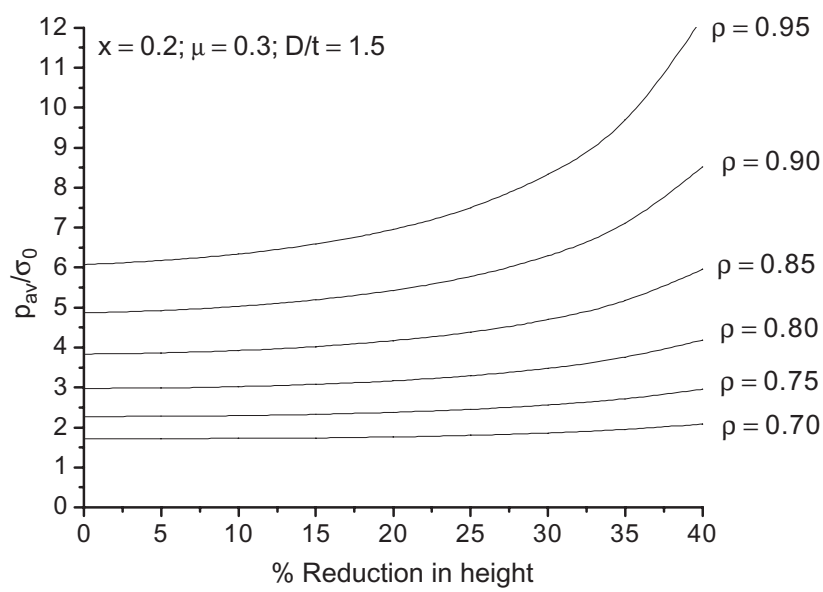

Figure 4. Variation of relative average pressure with percentage reduction in height of the hexagonal disc at different values of initial relative density.

The relative density of the preform increases with the forging pressure and it is asymptotic (figure 6), i.e. nearly equal to 1 . It is observed that the relative density of the preform increases sharply during initial phase of loading. It is due to the fact that an initially large amount of load is consumed in compaction and then, after gaining sufficient relative density, it is deformed and gives a uniform proportionate curve.

\section{Conclusion}

During forging of powder preforms, the mode of deformation is quite different from that of wrought materials and it is a function of both density and hydrostatic stress. In powder forging, mass constancy is to be assumed. During forging of metal powder preforms through closed dies, it is seen that compaction and compression both take place simultaneously. Initially the closing of pores dominates the compression process. The larger amount of applied load is utilized in densification and lesser amount is consumed for compression. Density distribution

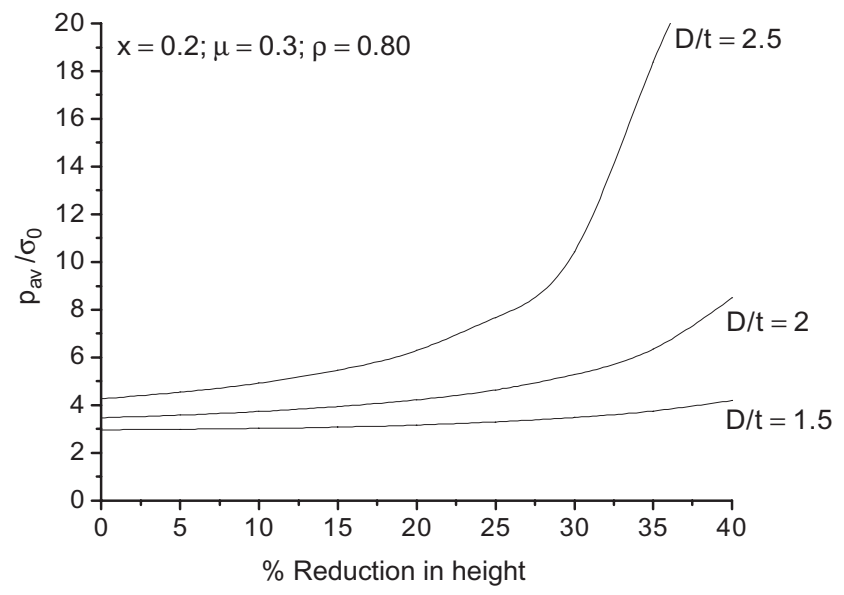

Figure 5. Influence of $D / t$ ratio on relative average pressure on the punch with percentage reduction in preform height. 


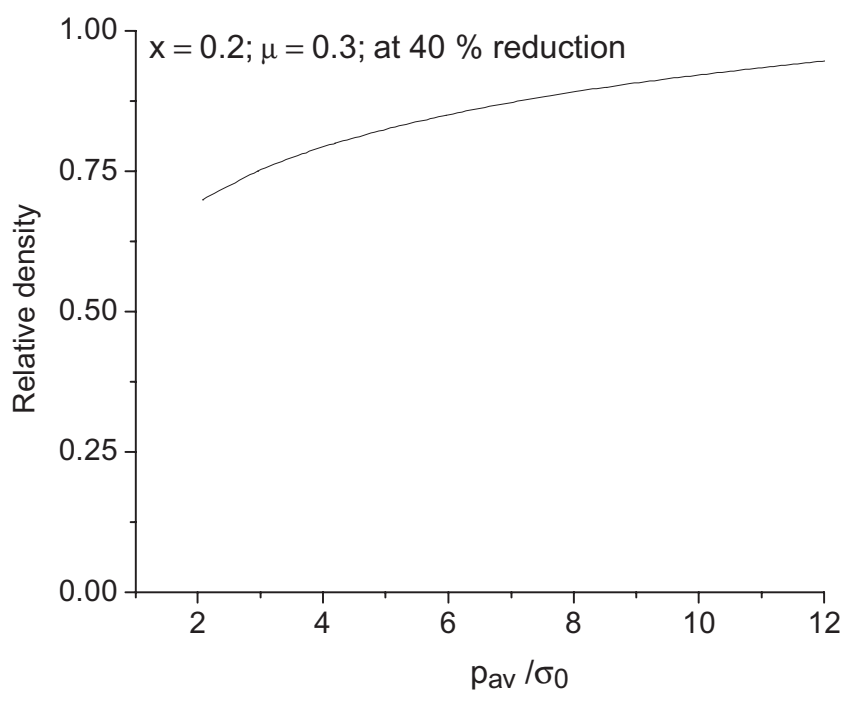

Figure 6. Variation of relative density with relative average pressure on punch.

is more uniform for a higher initial relative density of the preform. The density of the preform increases with increase in the forging load. If we can decrease the value of $D$ the side of the hexagonal nut, we can save on material as well as forging cost.

The above determined observations will be helpful to designing products of hexagonal shape, i.e. mainly hexagonal nuts. It gives guidelines for developing sintered products. These results can be expanded for other shapes. This solution can also be expanded with minor modification to the problem of forging of bolts.

This work would be effective for the determination of die loads during closed die forging of porous metals by the upper bound approach.

\section{Appendix A}

According to Tabata et al (1977) the principal strain increments are given as

$$
\mathrm{d} \varepsilon_{i}=\mathrm{d} \lambda\left(\frac{3\left(\sigma_{i}-\sigma_{m}\right)}{2 \sqrt{3} J^{\prime}} \pm \eta\right),(i=1,2,3),
$$

where

$$
\mathrm{d} \lambda=\frac{\sqrt{2}}{3}\left[\left(\mathrm{~d} \varepsilon_{1}-\mathrm{d} \varepsilon_{21}\right)^{2}+\left(\mathrm{d} \varepsilon_{2}-\mathrm{d} \varepsilon_{3}\right)^{2}+\left(\mathrm{d} \varepsilon_{3}-\mathrm{d} \varepsilon_{1}\right)^{2}\right]^{1 / 2} .
$$

The volumetric strain increment is given as

$$
\begin{aligned}
\mathrm{d} \varepsilon_{v} & =\mathrm{d} \varepsilon_{1}+\mathrm{d} \varepsilon_{2}+\mathrm{d} \varepsilon_{3}= \pm 3 \eta . \mathrm{d} \lambda \\
& = \pm \sqrt{2} \eta\left[\left(\mathrm{d} \varepsilon_{1}-\mathrm{d} \varepsilon_{2}\right)^{2}+\left(\mathrm{d} \varepsilon_{2}-\mathrm{d} \varepsilon_{3}\right)^{2}+\left(\mathrm{d} \varepsilon_{3}-\mathrm{d} \varepsilon_{1}\right)^{2}\right]^{1 / 2}
\end{aligned}
$$

For axisymmetrical compression:

$$
\sigma_{1}=\sigma_{3}=\sigma_{r}=\sigma_{\theta} \text { and } \sigma_{2}=\sigma_{\theta} .
$$


Hence,

$$
\mathrm{d} \varepsilon_{r}=\left[\frac{1}{2}-\eta\right] \mathrm{d} \lambda=\mathrm{d} \varepsilon_{\theta} \text { and } \mathrm{d} \varepsilon_{z}=-(1+\eta) \mathrm{d} \lambda .
$$

Putting the values from (A4) and (A5) into (A3) (considering the negative sign):

$$
2 \mathrm{~d} \varepsilon_{r}+\mathrm{d} \varepsilon_{z}=-2 \eta \mathrm{d} \varepsilon_{r}+2 \eta \mathrm{d} \varepsilon_{z} .
$$

Rearranging leads to

$$
\mathrm{d} \varepsilon_{r}=\frac{(2 \eta-1)}{2(1+\eta)} \mathrm{d} \varepsilon_{z} .
$$

Considering $\mathrm{d} \varepsilon_{z}=\mathrm{d} t / t$ and using it in (A7) gives

$$
\mathrm{d} \varepsilon_{r}=\frac{(2 \eta-1) \mathrm{d} t}{2(1+\eta) t}
$$

and upon integrating,

$$
\varepsilon_{r}=\frac{(2 \eta-1)}{2(1+\eta)} \log _{e} \frac{t_{2}}{t_{1}} .
$$

\section{List of symbols}

$D \quad$ sides of the hexagonal disc;

$k \quad$ constant equal to 2 in yield criterion;

$n \quad$ a constant quantity much greater than 1 ;

$t$ instantaneous thickness of preform;

$p_{a v} \quad$ ram pressure;

$r \quad$ radius of the generic point from origin at the corner;

$N \quad$ number of sides in a polygon;

$J_{2}^{\prime} \quad$ second invariant of deviatoric stress;

$\tau \quad$ shear stress;

$\mu \quad$ coefficient of friction;

$\lambda$ flow stress of metal powder preform;

$r, \theta, z \quad$ cylindrical co-ordinates;

$\rho \quad$ relative density of the preform;

$\sigma_{0} \quad$ yield stress of the non-work hardening matrix metal;

$\dot{\varepsilon}_{r}, \dot{\varepsilon}_{\theta}, \dot{\varepsilon}_{z}$ principal strain increment;

$\eta \quad$ constant and a function of $\rho$ only.

\section{References}

Deryagin B V 1952 What is friction? Iz d̆. Akad. Nauk. USSR

Jha A K, Kumar S 1983 Forging of metal powder preforms. Int. J. Mach. Tool Des. Res. 23: 201-206 Jha A K, Kumar S 1984 Forging of a hollow metal powder preform disc. Adv. Technol. Plast. Jpn. 1: 353-358 
Jha A K, Kumar S 1985 Analysis of axisymmetric cold processing of metal powder preforms. J. Inst. Eng. India 65: 169-174

Ramakrishnan P 1980 Forging of metal powder preforms. Proc. Int. Seminar on Metal Working Technology Today and Tomorrow, NIFFT, Ranchi, pp 43-46

Sagar R, Juneja B L 1980 An upper bound solution for closed die forging of hexagonal shapes. Int. J. Mach. Tool Des. Res. 20: 67-73

Tabata T, Masaki S 1975 Plane strain extrusion of porous materials. J. Jpn. Soc. Technol. Plast. 16: $279-284$

Tabata T, Masaki S, Hosokawa K 1980 A compression test to determine the coefficient of friction in forging P/M preforms. Int. J. Powder Metall. Powder Technol. 16: 149-155

Tabata T, Masaki S, Abe Y 1977 A yield criterion for porous metals and analysis of axial compression of porous discs. J. Jpn. Soc. Technol. Plast. 18: 373-380 